\title{
Philosophiques
}

\section{L'art in extremis: le monochrome chez Theodor W. Adorno et Yves Klein}

\section{Iain Macdonald}

Volume 33, numéro 2, automne 2006

URI : https://id.erudit.org/iderudit/013892ar

DOI : https://doi.org/10.7202/013892ar

Aller au sommaire du numéro

Éditeur(s)

Société de philosophie du Québec

ISSN

0316-2923 (imprimé)

1492-1391 (numérique)

Découvrir la revue

Citer cet article

Macdonald, I. (2006). L’art in extremis: le monochrome chez Theodor W. Adorno et Yves Klein. Philosophiques, 33(2), 455-471.

https://doi.org/10.7202/013892ar
Résumé de l'article

L'oeuvre de Theodor W. Adorno, et plus particulièrement sa Théorie esthétique, témoigne de sa défense soutenue de l'art moderne. Toutefois, dans le cadre de ses réflexions, on ne doit pas oublier qu'elle comporte également une dimension critique. Sa polémique à propos du jazz, par exemple, est devenue célèbre. Par contraste, sa critique de la peinture monochrome demeure relativement inconnue. Ce texte propose d'abord d'esquisser les éléments de celle-ci afin de tester ensuite ses limites en analysant une oeuvre monochrome d'Yves Klein: IKB 79. 


\title{
L'art in extremis: le monochrome chez Theodor W. Adorno et Yves Klein
}

\author{
IAIN MACDONALD \\ Université de Montréal \\ iain.macdonald@umontreal.ca
}

\begin{abstract}
RÉSUMÉ. - L'œuvre de Theodor W. Adorno, et plus particulièrement sa Théorie esthétique, témoigne de sa défense soutenue de l'art moderne. Toutefois, dans le cadre de ses réflexions, on ne doit pas oublier qu'elle comporte également une dimension critique. Sa polémique à propos du jazz, par exemple, est devenue célèbre. Par contraste, sa critique de la peinture monochrome demeure relativement inconnue. Ce texte propose d'abord d'esquisser les éléments de celle-ci afin de tester ensuite ses limites en analysant une œuvre monochrome d'Yves Klein : IKB 79 .
\end{abstract}

\begin{abstract}
Theodor W. Adorno's defence of modern art in Aesthetic Theory and other writings is well known, as is his scathing attack on jazz. Less well known is his critique of monochromatic painting. This article first sketches the stakes and terms of this critique in relation to Clement Greenberg's dismissal of much monochromatic or near-monochromatic painting, before moving on to an analysis of Adorno's position. For the purposes of this analysis, Yves Klein's IKB 79 serves as a test case.
\end{abstract}

Dans la résistance au monde fongible de l'échange, est irréductible celle du regard qui ne veut pas que le monde perde toutes couleurs. ADORNO, Dialectique négative

Certaines tendances de l'art moderne, dont celles de la peinture monochrome et de l'abstraction radicale, sont parfois reçues avec hésitation, si ce n'est avec scepticisme. Est-ce de l'art ou non ? Si c'en est, ne serait-ce pas simplement du mauvais art? Ces questions ne s'expliquent pas par un simple manque de culture ou par l'attitude relâchée qui présuppose que l'art est ce qui se trouve accroché aux murs du musée. Dans une perspective formaliste, Clement Greenberg, par exemple, a notoirement rejeté la plupart de la peinture monochrome en invoquant son caractère arbitraire ainsi que la surenchère de l'abstraction qui conduit la peinture à perdre sa capacité à formuler quoi que ce soit.

Ainsi, Greenberg considère que, loin d'avoir cristallisé l'idéal moderniste, les tendances les plus extrêmes de l'évolution de la peinture vers l'abstraction et le monochrome au milieu du $\mathrm{XX}^{\mathrm{e}}$ siècle l'ont vicié. La réduction tout à fait légitime de la peinture à ses composantes les plus fondamentales (à savoir, selon Greenberg, la forme de la toile, la planéité de sa surface et le pigment) serait 
allée trop loin dans la peinture monochrome parce qu'elle transforme une purification créative de la peinture en une évacuation de possibilités expressives. En ce sens, l'art se voit menacé d'un danger pressant: les peintures monochromes seraient trop « arbitraires ", trop « sculpturales » ou trop « chosiques » - ce qui, en fin de compte, signifie qu'elles courent le risque de compromettre toute distinction entre l'art et le non-art. En appréhendant inadéquatement et en exagérant l'histoire interne du haut-modernisme, en transformant la tendance légitime vers une purification du médium en un débordement dans le domaine du non-art, les œuvres monochromes traquent aveuglément les éléments premiers de la peinture (ou de l'art en tant que peinture) au point où elles sombrent dans le non-art ou — dans le meilleur des cas - du mauvais art: une œuvre authentiquement monochrome, de dire Greenberg, est indiscernable d'une simple toile tirée, montée et apprêtée. Bien que Greenberg soit considéré, à juste titre, comme l'un des défenseurs les plus hardis de la peinture moderniste, sa réaction au monochrome témoigne de son exaspération face à ce dépérissement de l'art :

J'ai été surpris de voir à quel point [les peintures blanches et noires de Rauschenberg] étaient faciles à "saisir ", à quel point elles avaient quelque chose de familier et de superficiel [slick] même. Ça n'a pas été différent par la suite quand j'ai vu les monochromes, ou quasi-monochromes, de Reinhardt, Sally Hazlett et Yves Klein. [...] Une surface plane monochromatique, qu'on pouvait voir comme une étendue limitée et différente d'un mur, élève désormais cette prétention d'être automatiquement un tableau, de l'art ${ }^{1}$.

Comme nous le verrons plus loin, Theodor Adorno est également assez peu sympathique aux œuvres modernes les plus extrêmes, mais sa perspective théorique et critique va plus loin que les considérations formalistes de Greenberg. La question qui nous occupera est la suivante : dans son antipathie, Adorno ne sous-estime-t-il pas la capacité d'expression du monochrome ? À cela ajoutons déjà cette précision, à savoir que la réponse que nous formulerons à cette critique sera tout aussi adornienne que la question qui l'a suscitée.

La théorie esthétique d'Adorno se développe autour d'une analyse de l'expérience esthétique qui garde l'œuvre concrète à portée de vue et qui situe ainsi la vérité de l'art dans un certain mouvement commun à l'œuvre et à l'expérience esthétique: "la théorie de l'art, affirme-t-il, n'a pas à se situer audelà [de l'art] mais doit s'abandonner aux lois de son mouvement ${ }^{2}$, de

1. Clement Greenberg, "Recentness of Sculpture " in Clement Greenberg, The Collected Essays and Criticism, sous la direction de J. O'Brien, 4 tomes (Chicago : University of Chicago Press, 1993), t. 4, p. 251. Sur Greenberg et la peinture monochrome, voir Thierry de Duve, Kant after Duchamp (Cambridge, MA: The MIT Press, 1996), pp. 199-280.

2. Theodor W. Adorno, Théorie esthétique. Paralipomena, Théories sur l'origine de l'art, Introduction première, trad. Marc Jimenez et Éliane Kaufholz, nouvelle édition revue et corrigée (Paris : Klincksieck, 1989, 1995), p. 183. Gesammelte Schriften [GS], sous la direction de Rolf Tiedemann, 20 tomes (Frankfurt am Main: Suhrkamp, 1997), t. 7, p. 194. 
sorte que la vérité de l'art n'apparaisse que dans l'expérience esthétique, à savoir dans la reconnaissance des lois énigmatiques immanentes à l'œuvre singulière. Mais cela n'est pas toujours facile, car l'œuvre d'art ne se laisse pas interpréter immédiatement : "Les œuvres d'art sont énigmatiques en tant que physionomie d'un esprit objectif qui n'est jamais transparent à soi-même au moment de son apparition ${ }^{3}$." Autrement dit, les œuvres d'art sont physionomiques en ce sens qu'elles sont, chacune à sa façon, clairement saturées d'une dimension spirituelle et expressive : dans la constellation d'éléments qui constituent l'œuvre, il se trouve des lois d'expression uniques à partir desquelles celle-ci semble exprimer quelque chose d'humain - son objectivité signifie et, en vertu de cette signification apparente, demande à être interprétée. Mais, en même temps, ces « lois du mouvement » immanentes de l'œuvre d'art sont telles que cette signification ne pourra jamais être claire et distincte; elles ne sont jamais (du moins en ce qui concerne les œuvres réussies) susceptibles d'être déchiffrées " une bonne fois pour toutes ", bien que l'on doive comprendre l'œuvre comme une source de normativité qui guide l'interprétation. Le va-et-vient entre sujet et objet, interprète et œuvre, ne peut être supprimé parce que la possibilité d'une interprétation ultérieure, divergente mais cohérente, empêche toute compréhension finale et totalisante. Parler de la physionomie énigmatique de l'œuvre d'art est donc une façon de résumer sa capacité particulière d'expression.

Ainsi, parce qu'elle reconnaît cette exigence d'interprétation, difficile et parfois frustrante, présente dans toute œuvre authentique, la pensée cherche à comprendre l'œuvre et l'art en tant que tels (même la question familière " estce de l'art? " implique la question «qu'est-ce que l'art ? »). Par conséquent, l'interprète n'y répond pas par une subsomption de l'œuvre concrète dans l'esprit subjectif ou par un simple acte classificatoire (par exemple, "c'est un tableau »); il doit trouver les concepts qui conviennent à l'expérience esthétique particulière sans détourner les yeux de l'œuvre, tout en admettant qu'une explication compréhensive ne peut jamais émerger de cette expérience : "L'expérience artistique exige un comportement cognitif et non une réaction affective à l'égard des œuvres ${ }^{4}$. » Face à l'œuvre singulière, à la norme particulière qu'elle exprime de manière ambiguë, l'interprétation doit s'avérer aussi singulière que l'œuvre, bien que l'on sache qu'aucune œuvre n'est transparente au point qu'une seule interprétation puisse la capturer définitivement ${ }^{5}$. Comme l'affirme Adorno, il y a des «moments de l'art irréductibles au sujet, impossibles à posséder dans leur pure immédiateté ${ }^{6}$ ».

3. Adorno, Théorie esthétique, p. 183. GS, t. 7, p. 194.

4. Adorno, Théorie esthétique, p. 492 . GS, t. 7, p. 528.

5. Ces « singularités en parallèle ", l'idée que l'interprétation doit s'avérer aussi singulière que l'œuvre déterminée par l'interprétation ouvre une piste pour comprendre pourquoi Adorno emploie le terme mimesis pour caractériser le rapport entre l'identité et la non-identité.

6. Adorno, Théorie esthétique, p. 489 . GS, t. 7, p. 524. 
Autrement dit, la vérité de l'art repose à la fois sur sa normativité ambiguë et sur l'interprétation qui répond à cette normativité. De la même manière, Adorno sépare aussi le contenu de l'œuvre d'art des thèmes et des intentions subjectives spécifiques qui pourraient s'y manifester. Ainsi, les moments de l'œuvre «irréductibles au sujet, impossibles à posséder dans leur pure immédiateté ", proviennent précisément de la dialectique immanente propre à l'expérience esthétique, c'est-à-dire de la médiation de l'esprit et de la matière; ils ne peuvent être réduits aux intérêts universels d'un contenu thématique spécifique quelconque, que ce soit celui d'un récit, d'un sujet ou d'un objet représenté. Dans le cas d'œuvres d'art très abstraites, c'est clair : elles semblent signifier, mais leur signification ne peut être saisie sans équivoque par le biais d'une explication unique. Elles signifient, mais de façon ouverte, souvent parce qu'elles refusent toute dépendance à un contenu thématique. Cette capacité apparente à signifier énigmatiquement, indépendamment de toute objectivité représentée, constitue ce que Adorno appelle l'apparence esthétique (Schein). La vérité de l'art, ou la substance de l'œuvre, réside alors dans cette apparence, car elle ne peut résider ni dans la forme, ni dans un contenu thématique quelconque, ni dans une technique pré-établie qui lie ceux-là ensemble (une méthode mécanique de production, par exemple). En d'autres termes, elle réside dans une relation irréductible qui lie la pensée et l'être, l'esprit et la nature - non pas en tant que contraires mis en relation, mais plutôt en tant que dimensions essentielles de l'expérience qui, toujours, s'interpénètrent.

Par conséquent, l'apparence esthétique est à comprendre à partir de la signification, à partir du caractère «interpellant » de l'art et de la manifestation énigmatique de l'esprit dans l'ipséité de l'œuvre. Celle-ci nous donne une norme faible, pour ainsi dire, dans la mesure où la pure invention n'a pas sa place dans la réponse qu'elle exige de l'interprétation: elle doit nous guider, même si elle reste muette face à nos tentatives de compréhension. En ce sens, l'apparence de l'art est totalement immanente aux œuvres individuelles et à leur caractère historique; elle ne repose pas, par exemple, sur un idéal représentationnel. Cela conduit Adorno à lire l'esthétique de la forme et du contenu comme désespérément limitée:

Le contenu immanent des œuvres, leurs matériaux, et ses mouvements, sont fondamentalement différents du contenu en tant que détachable de l'intrigue d'une pièce de théâtre ou du sujet d'un tableau, tels que Hegel, en toute innocence, les identifie. [...] Le contenu d'un tableau n'est pas seulement ce qu'il représente, mais tout ce qu'il contient d'éléments de couleur, de structures, de rapports; le contenu d'une musique, par exemple, est — selon Schönberg — l'histoire d'un thème. L'objet [représenté] peut lui aussi compter comme élément, de même en littérature, l'action ou l'histoire narrée; mais ne compte pas moins tout ce qui se passe dans l'œuvre, ce par quoi elle s'organise, ce par quoi elle se modifie ${ }^{7}$.

7. Adorno, Théorie esthétique, pp. 493-494. GS, t. 7, p. 529. 
Dès lors, nous ne pouvons en rester avec le schéma général des formes d'art modelant un contenu thématique. Forme et contenu sont déjà inextricablement liés dans l'articulation et l'arrangement des matériaux «bruts"; la matière spiritualisée fait partie du contenu de l'œuvre et elle est bel et bien distincte de n'importe quel contenu thématique de celle-ci. Dans un paysage, la traduction de la nature sur la toile peut être enregistrée de diverses façons et à divers degrés selon la technique employée : dans le cas d'un tableau de Gerhard Richter, par exemple, ce sera par des coulées ou des traînées de peinture, ou bien par un effet de flou. Ces effets ne sont nullement secondaires; au contraire, la manière dont Richter rend la nature fait explicitement partie de la construction de l'œuvre; ce qu'il rend est secondaire et doit se comprendre uniquement selon le «comment » de son apparence. De même, dans plusieurs tableaux de Mondrian, où des lignes superposées définissent des carrés monochromes de couleurs primaires, la réalité est rendue selon des opérations quasigéométriques. New York City (1942), par exemple, est moins un paysage urbain (sans que cette signification ne soit pour autant perdue) que la présentation unique d'une règle subjective de l'abstraction, liée rigoureusement et étroitement à la réalité dont l'œuvre est dérivée ${ }^{8}$. La nature et le contenu représentationnel deviennent des moments évanescents dans l'art abstrait, proportionnellement à l'élaboration des lois internes du médium et du processus spirituel par lesquels l'œuvre vient au jour'.

Ainsi, il semble naturellement découler de la thèse d'Adorno que l'art abstrait tend à être "plus vrai », pour ainsi dire, que l'art représentationnel. Après tout, une fois la nécessité du contenu thématique compromise, une fois libéré des considérations dérivées ou accessoires du médium artistique spécifique, l'art est libre d'explorer ses structures internes. On peut dès lors concevoir l'art abstrait comme s'étant émancipé de la représentation et des contraintes arbitraires, comme ayant découvert son "contenu immanent", à savoir, tous ces "éléments de couleur, de structures, de rapports » qu'une œuvre contient. À cet égard, la théorie d'Adorno peut même ressembler à celle de Greenberg.

En effet, Adorno semble défendre l'abstraction comme la manière même dont les œuvres d'art deviennent «modernes» et conscientes de leur apparence ainsi que de l'indétermination du sens qui les caractérise:

Se défaire des intentions dans la production des œuvres — « faire en sorte que l'on ne puisse dire ce que sont les choses » — prête à l'œuvre le caractère de signe.

8. Par « règle subjective » nous entendons ce en vertu de quoi on peut dire que New York City est une œuvre de Mondrian, en dehors de considérations para-artistiques (une signature ou une déclaration verbale provenant de l'artiste, par exemple).

9. Bien entendu, Hegel tient compte de cet aspect de la peinture dans ses leçons sur l'esthétique, mais il s'agit toujours pour lui d'une transformation subjective de l'objet représenté; il n'aurait jamais prévu la "perte» de l'objet qui caractérise les formes les plus poussées de l'art moderne. 
Elle devient un signe précisément à travers une rupture entre elle-même et tout signifié. L'écriture en musique et en peinture [c'est-à-dire l'apparente élaboration du sens], toutefois, n'est pas directement comme un script; elle ressemble plutôt à un script encrypté. Autrement, l'œuvre reste sur le plan de l'imitation. L'écriture est par conséquent d'essence historique: elle est moderne. Elle est libre en vertu de ce que nous appelons - selon une expression malheureuse - l'abstraction, c'est-à-dire, un certain refus de l'objectivité [Gegenständlichkeit ${ }^{10}$.

Or on pourrait bien être tenté, à partir de ce passage, de conclure que le contenu d'une œuvre n'a pas besoin d'être autre chose que les "éléments de couleur, de structures, de rapports ». Car si l'histoire de l'art du XX $\mathrm{XX}^{\mathrm{e}}$ siècle est bien la preuve de quelque chose, semble-t-il, alors elle est la preuve que le contenu en tant que sujet représenté est à concevoir comme secondaire par rapport à la constellation des matériaux de l'œuvre. On peut même envisager qu'il serait possible de se dispenser complètement du sujet portraituré, exception faite de ce sujet primitif qui concerne le médium et la disposition expressive de ses matériaux. Évidemment, dans le contexte d'un tel concept d'abstraction, le monochrome n'est plus la seule figure d'intérêt: Malevitch, le constructivisme russe, et même Kandinsky peuvent tous être cités comme des exemples préalables d'une extirpation du contenu thématique explicite. Et, en effet, Adorno approuve clairement la perte de contenu thématique ou, plutôt, l'accent désormais mis sur la constellation immanente du matériel artistique, du moins dans la mesure où le résultat de l'abstraction demeure expressif:

Les tendances [artistiques] qui depuis Kandinsky ont été conçues sous le nom d'art abstrait sont seulement abstraites dans la mesure où elles se sont détournées des objets manifestes; par contre, en tant que formations artistiques [Gestaltungen], elles doivent subir le même standard de concrétion que tout $\operatorname{art}^{11}$.

Bref, l'art abstrait semble bénéficier d'un certain avantage sur l'art figuratif dans la mesure où l'absence d'objets laisse apparaître l'apparence en tant que telle, c'est-à-dire, la construction ou la formation de matériaux dans une structure concrète, expressive, et non objective. De cette façon, la « fin » de l'art dans le cadre de l'abstraction semble consister en une sorte de purification de l'apparence esthétique et en une réduction aux éléments les plus fondamentaux de la pratique artistique. Prenons, à titre d'exemple extrême de cette purification, les tableaux aux couleurs pures de Rodchenko,

10. Theodor W. Adorno, «Über einigen Relationen zwischen Musik und Malerei » in $G S$, t. 6, p. 634. Le mot «écriture" paraît en français dans le texte.

11. Theodor W. Adorno, "Abstrakt oder konkav?" in GS, t. 20, p. 522. Mais Adorno se méfie, bien entendu, du spiritualisme de Kandinsky: « dans sa révolte motivée contre un sensualisme qui, jusque dans le Jugendstil, accorde à la satisfaction sensible en art une place prépondérante, il isolait abstraitement l'opposé de ce principe et le réifiait : il était ainsi difficile de distinguer le "tu dois croire à l'esprit", de la superstition et de l'exaltation des «choses supérieures» à laquelle se livrait le style art déco ». (Adorno, Théorie esthétique, p. 130. GS, t. 7, pp. 134-135.) 
qui furent exposés pour la première fois en 1921 et auxquels l'histoire de l'art fait l'honneur de les considérer comme les premières vraies œuvres monochromes de l'ère moderne ${ }^{12}$. Rodchenko a prétendu avoir amené la peinture à sa «conclusion logique» en la réduisant à sa forme la plus pure par l'application d'un pigment sur une surface finie. Disposées les unes à côté des autres, ces œuvres constituent le paradigme de la pureté réductrice. Rodchenko écrit: "J'ai réduit la peinture à sa conclusion logique en exposant trois toiles: une rouge, une bleu, et une jaune. J'ai affirmé: voilà, c'est fichu! Des couleurs primaires. Chaque plan est un plan et il n'y aura plus de représentation ${ }^{13}$. » Bien sûr, la représentation était dépassée seulement dans le sens étroit du terme; elle persistait historiquement sous une forme altérée en tant que la présentation oblique de la représentation elle-même. Quant à Rodchenko, après l'exposition de ses monochromes, il a renoncé tout à fait à la peinture, convaincu que son potentiel avait été épuisé.

Et pourtant, contrairement à nos attentes peut-être, Adorno n'accepte pas la réduction de l'art à ses composantes matérielles. Ainsi, plutôt que d'embrasser les formes les plus extrêmes de l'abstraction dans les arts, lesquelles revendiquent pourtant le statut de structures purement expressives de l'apparence esthétique, il rejette toute réduction formelle au médium. Qu'est-ce donc qui ne va pas quant au monochrome, selon Adorno ? En bref: il risque de réduire l'art au statut d'une simple chose pré-artistique et, ce qui est plus grave, d'atténuer l'enchevêtrement crucial de l'idéalité et de la réalité, de la matière et de la forme, qui est au cœur de la production artistique et de l'expérience esthétique ${ }^{14}$.

Dans cette quête de pureté, dans cette tentative d'isoler ce qui est réellement «artistique » dans l'art, de telles œuvres courent le risque d'anéantir celuici et de ne laisser qu'un grand nombre d'objets insignifiants, voire de toiles «incomplètes », seulement tirées, montées et apprêtées. Effectivement, la tradition du monochrome, c'est-à-dire ce courant de la pratique artistique moderne qui a poursuivi l'idéal de l'abstraction jusqu'à sa conclusion logique, serait, selon Adorno, un excellent exemple d'art qui s'anéantit de cette manière. Ainsi, comme il l'affirme, "dès que l'œuvre d'art éprouve des craintes aussi fanatiques pour sa pureté au point de s'y perdre elle-même et de tourner vers l'extérieur ce qui ne peut plus devenir art (toile et matériau sonore brut), elle devient son propre ennemi ${ }^{15}$.» En d'autres termes, et par

12. Voir cependant Denys Riout, "La peinture monochrome: une tradition niée " in Les cahiers du Musée national d'art moderne, $\mathrm{n}^{\circ} 30$ (hiver 1989): pp. 81-98.

13. Il semble que cette citation marquante soit tirée d'un texte de Rodchenko sur Maïakovski, datant de 1939. La référence présente est à Anna Moszynska, «Purity and Belief: The Lure of Abstraction " in The Age of Modernism: Art in the Twentieth Century (Stuttgart et Berlin: Verlag Gerd Hatje \& Zeitgeist-Gesellschaft, 1997), p. 204.

14. Adorno parle de la régression vers "une choséité néfaste [schlechte Dinghaftigkeit]" ou vers « la pure et simple chosalité [bloße Dinghaftigkeit]». Voir Adorno, Théorie esthétique, pp. 64 et 150. $G S$, t. 7 , pp. 63 et 158.

15. Adorno, Théorie esthétique, p. 150-151. GS, t. 7, p. 158. Traduction modifiée. 
rapport à Rodchenko (entre autres), cette entreprise visant à créer un art "pur » peut aboutir à une simple forme de réductionnisme: une peinture dépouillée de toute fonction picturale, de contrastes de couleurs et de lignes, n'est pas pour autant une bonne œuvre d'art. En fait, elle sera presque nécessairement une mauvaise œuvre d'art parce qu'elle confond irrémédiablement les éléments fondamentaux du médium avec la pureté ou l'essence: moyens et fins se confondent, ce qui revient à une "continuation directe et fausse de la rationalité des fins ${ }^{16}{ }$. Pour Adorno, la soi-disant «pureté » artistique ne peut s'exprimer que sous le mode d'une lutte interprétative entre la matérialité et l'idéalité. L'art émane de cette lutte; il ne se constitue pas en proportion de la réduction d'un médium à ses composantes fondamentales. Pour le dire de façon concise: "À l'inverse de ce que veut le cliché, l'art moderne n'est pas débile lorsqu'il va trop loin, selon cette même phraséologie, mais lorsqu'il n'est pas allé assez loin, lorsque les œuvres vacillent par manque de rigueur logique ${ }^{17}$.» $\mathrm{Ou}$ encore :

Si la peinture, ou la musique, en venait simplement à perdre son moment expressif, le moment de l'expression ou de ce qui est à exprimer - si l'œuvre n'était plus en tension avec quelque chose d'autre que son propre phénomène, quelque chose qui n'est ni dissimulé en elle en tant qu'unité symbolique ni situé à l'extérieur d'elle, alors son caractère d'écriture serait perdu. L'œuvre régresserait (comme plusieurs aujourd'hui) vers le pré-artistique ${ }^{18}$.

Dans ce contexte, semble-t-il, le monochrome ne peut qu'échouer: l'objet d'art supposé qui se rapproche de l'objectivité générique par le biais d'un processus formel de soustraction risque de perdre la tension nécessaire entre matière et idée. L'essence de l'art s'éloigne du domaine de la raison et de la connaissance pour passer à celui des blagues, des curiosités, et du bricà-brac ${ }^{19}$. En ce qui concerne la peinture monochrome, elle est guidée par les idéaux abstraits de la réduction à la couleur pure et de la pureté du médium en se manifestant dans un objet qui, par sa simplicité, pourrait très bien ne pas être de l'art (une simple toile apprêtée, par exemple). Ou encore, du côté de l'interprète, on pourrait dire que l'interprétation d'une œuvre ne devrait pas simplement aboutir à l'acte de comprendre le but abstrait de l'autoréduction de la peinture à ses composantes essentielles, c'est-à-dire à l'acte de "piger ", de saisir intelligemment le processus de soustraction qui aboutit à la peinture monochrome. On devrait plutôt en venir à une sorte d'équilibre

16. Adorno, Théorie esthétique, p. 151. GS, t. 7, p. 158.

17. Adorno, Théorie esthétique, p. 60. GS, t. 7, p. 58.

18. Adorno, «Über einigen Relationen zwischen Musik und Malerei », GS, t. 16, p. 635.

19. Il ne s'agit pas seulement de la peinture monochrome, mais très certainement aussi de l'art conceptuel. En effet, de celui-ci on peut dire que le caractère conceptuel de l'œuvre prime sur sa matérialité : l'aspect sensible est purement transitoire. Du coup, la peinture monochrome ferait pendant à l'art conceptuel en ce sens que, dans son cas, c'est la matérialité qui prime sur l'idéalité, laquelle est presque éliminée de l'œuvre. Dans les deux cas, par conséquent, il s'agirait selon Adorno d'une régression vers le pré-artistique. 
précaire entre le désir de rendre justice à l'œuvre individuelle qui suscite la réflexion et l'inadéquation inévitable (quoique éventuellement féconde) de tout énoncé que l'on prononce à son sujet. C'est ce que Adorno appelle la transcendance ou le caractère énigmatique de l'œuvre. Celle-ci ne devrait pas se désintégrer d'emblée une fois qu'une interprétation est proposée : elle doit pouvoir persister en tant que potentiel objectif et en tant que référent de futures interprétations, c'est-à-dire, comme une source durable de normativité. Sa vérité émerge d'actes d'interprétation qui aident à la constituer et non du jugement subsumant qui assimile la méthode employée avec l'essence de l'œuvre. En résumé, donc, l'art monochrome s'abandonnerait à la barbarie : "Les avancées esthétiques les plus extrêmes s'emmêlent avec la régression. L'avenir de l'art dépend de ce que le progressif en lui soit de taille à lutter contre le régressif, sinon il succombera à cette littéralité barbare qui règne aujourd'hui dans le culte des méthodes et des matériaux absolus ${ }^{20}$.»

Pourtant, cette critique n'est peut-être pas aussi convaincante qu'elle le voudrait. Elle exige de ses tenants cette supposition fondamentale que les œuvres monochromes dépendent toutes, et dans une large mesure, de moyens formels qui abolissent toute possibilité expressive. C'est ainsi, peut-être, que le geste de Rodchenko demande à être interprété, lui qui, selon ses propres dires, a poussé la peinture à sa conclusion logique; par rapport à ce type d'œuvre, la salve d'Adorno semble bien atteindre la cible. Mais dans d'autres cas, et en particulier pour ce qui est de la peinture monochrome après Rodchenko, là où l'accusation parle de la réduction de l'art à ses propriétés sensibles insignifiantes, on découvre pourtant certaines œuvres tout aussi monochromatiques que celles de Rodchenko qui semblent échapper à la critique.

Force est d'admettre, tout d'abord, que les œuvres monochromes ne sont pas toutes également intéressantes. Apparaît ainsi une différenciation entre ces œuvres plus significative que celle qu'Adorno admet explicitement. Les peintures bleues de Klein ne sont-elles pas aussi éloignées des peintures blanches de Rauschenberg que les œuvres de Lucio Fontana le sont de celles de Piero Manzoni ? Cela ne suggère-t-il pas déjà qu'il s'agit d'un phénomène plus nuancé que celui qui est critiqué et rejeté par Adorno ? La prochaine question qui se pose est donc celle-ci : l'élimination de la ligne picturale et de la

20. Adorno, «Über einigen Relationen zwischen Musik und Malerei », GS, t. 16, p. 642. La régression dont parle Adorno ne cible pas seulement les éléments " désuets » dans l'œuvre qui se veut moderne, c'est-à-dire des éléments qui ont leur origine dans des formes antérieures dont l'artiste dépend à son insu ou de manière non critique. Selon Adorno, la régression ne se manifeste pas seulement là où la critique est absente, mais partout où l'expérience esthétique succombe à des exigences non esthétiques (à la production industrielle de biens culturels, par exemple). C'est ainsi qu'Adorno présente la régression dans «Über den Fetischcharakter in der Musik und die Regression des Hörens » in $G S$, t. 14, p. 14-50. Dans le contexte qui nous occupe, la régression revêt le caractère spécifique du "culte des méthodes et des matériaux absolus ", c'est-à-dire la réduction au pré-artistique (en ce qui concerne le monochrome: le support et la couleur pure). 
palette multicolore signifie-t-elle également, et nécessairement, l'élimination de l'art lui-même ${ }^{21}$ ?

Évidemment, il serait contre-productif, voire impossible, de défendre la peinture monochrome dans tous ses registres. Bien qu'il nous mène certainement dans la direction de l'abstraction totale et d'une perte de signification, le corpus des œuvres monochromes ne peut être écarté d'emblée par une approche interprétative quelconque, qu'elle soit de nature formaliste (Greenberg) ou philosophique (Adorno). Si tel était le cas, évidemment, les œuvres en question feraient toutes partie d'une classe générale d'objets indifférents dépendant tous de la même idée réductionniste. On pourrait alors les classer tous sous la même rubrique et les distinguer les uns des autres seulement arbitrairement ou en vertu de leurs qualités physiques. Dans ce cas, l'art régresserait véritablement vers le non-art. Par contre, nul n'est obligé d'accepter ce verdict tel quel.

Yves Klein est un artiste qui se démarque dans l'histoire de la peinture monochrome. Ses œuvres, bien qu'elles ne soient pas sans précédents dans le genre (Rauschenberg le devance de quelques années, et le geste nihiliste de Rodchenko demeure toujours le premier acte de réduction minimaliste de la peinture moderne à ses éléments fondamentaux), demeurent parmi les plus impressionnantes, tant par leur variété qu'en raison de l'utilisation idiosyncrasique de ce bleu qui a fait sa marque. Le corpus de Klein, développé dans l'essor du minimalisme et de l'art conceptuel, représente l'une des explorations les plus poussées du monochrome dans l'histoire de l'art. Et pourtant, bien qu'incontestablement réductrices, et pour des raisons qui devront être développées, ses œuvres monochromes résistent à des attaques comme celles d'Adorno. Bref, si nous choisissons Klein pour mettre à l'épreuve la critique adornienne de la peinture monochrome, c'est principalement pour deux raisons. Historiquement, Klein est sans doute l'artiste le plus important dans l'histoire du monochrome ou, du moins, celui qui a porté le plus loin sa passion pour la couleur pure. Mais, thématiquement, nous verrons qu'en raison de sa façon de «spiritualiser » ses matériaux, Klein est l'artiste dont la production monochrome répond le plus nettement à la critique énoncée par Adorno ${ }^{22}$.

L'intérêt de Klein pour la couleur pure est déjà évident dans ses œuvres de la première heure et se poursuit tout au long de sa brève carrière sous différentes formes: des pièces monochromes en orange, or, rose, et tout spéciale-

21. Même si Adorno critique la réduction au pré-artistique opérée par la peinture monochrome, il paraît à première vue plus ouvert à la réduction au noir comme «idéal » moderne : le noir signifie résistance à la consolation. Mais, précisément, en tant qu'idéal, le noir finit par se falsifier: "L'art dénonce la pauvreté inutile par celle qui lui est propre et volontaire, mais il dénonce aussi l'ascétisme et ne peut se contenter de l'ériger en norme. " (Voir Adorno, Théorie esthétique, pp. 66-68. GS, t. 7, pp. 65-67.)

22. Nous visons ce que Klein fait explicitement et comme l'un des premiers, sinon le premier, à faire ressortir le potentiel expressif de la couleur pure. Il n'est pas exclu que d'autres artistes puissent également réussir à répondre au défi lancé par la critique adornienne du monochrome. 
ment en bleu; des sculptures monochromes statiques et kinésiques; des tableaux en feu; des portraits relief; des "anthropométries »; et des reliefs éponges. Klein est bien sûr célèbre pour son bleu ultramarin, sa marque de commerce appelée IKB ou «International Klein Blue » qu'il a fait breveter en 1960. Aussi étrange que cela puisse paraître, la loi des brevets a permis à Klein de revendiquer cette couleur comme son invention protégée en lui accordant le brevet, non pas pour la couleur elle-même, mais pour la composition chimique de la combinaison du pigment et du solvant requis pour l'application de la couleur sur une surface. Heureux stratagème, donc, par lequel Klein aura indirectement réussi à faire breveter son bleu!

Que peut-on dire à propos d'un monochrome IKB ? De prime abord, très peu de choses, semble-t-il, sinon que "c'est bleu ». Cependant, par son utilisation de l'IKB, Klein a voulu nous présenter ce qu'il appelle la "matière liberté impalpable à l'état non concentré, non contracté ${ }^{23}$ ". La matière, en d'autres termes, qui, dans sa transformation artistique, résiste à une interprétation conceptuelle transparente et qui demeure indifférente aux vagues noms de couleurs dont on fait usage pour la désigner. À vrai dire, c'est seulement de la couleur IKB que l'on y voit et rien d'autre (mais dans ce cas, il faut avouer que l'on ne peut pas aller très loin en l'absence d'un des monochromes comme référent, ce qui signale déjà l'intérêt particulier de Klein). Cette réduction à la couleur pure était la passion de Klein en tant qu'artiste; c'était pour lui une tentative de libérer la couleur de la ligne: "Je suis contre la ligne et toutes ses conséquences : contours, formes, composition. Tous les tableaux, quels qu'ils soient, figuratifs ou abstraits me font l'effet d'être des fenêtres de prison dont les lignes, précisément, seraient les barreau ${ }^{24}$. » Mais lorsque la peinture commence à se libérer de la ligne, il devient de plus en plus difficile de décrire ce que c'est, ce qu'elle cherche à signifier. C'était en partie l'effet voulu : «au loin dans la couleur, dans la dominante, dans la liberté! Le lecteur d'un tableau à lignes, formes, composition, reste prisonnier de ses cinq sens ${ }^{25}$." Rodchenko a désespéré du nihilisme inhérent à cette provocation et a abandonné la peinture. Klein, quant à lui, a essayé de préserver quelque chose en purgeant la peinture de la ligne et, du coup, apparemment, du contenu.

En tirant au clair l'expressivité et l'apparence esthétique de l'œuvre de Klein, nous allons porter notre attention sur un seul tableau monochrome, IKB 79 , qui date de $1959^{26}$.

23. Yves Klein, "L'aventure monochrome" in Yves Klein. La Vie, la vie elle-même qui est l'art absolu, sous la dir. de Gilbert Perlein et Bruno Corà (Nice: Musée d'art moderne et d'art contemporain de Nice, 2000), p. 78. Évidemment, les déclarations de Klein ne sont pas dotées d'une autorité spéciale par rapport à d'autres interprétations, mais on peut très bien les évaluer au même titre que d'autres interprétations, c'est-à-dire à l'aune des œuvres.

24. Yves Klein, "Le dépassement de la problématique de l'art» in Yves Klein. La Vie, la vie elle-même qui est l'art absolu, p. 71.

25. Klein, "Le dépassement de la problématique de l'art", p. 71.

26. Pigment pur et résine synthétique sur bois, $1397 \mathrm{~mm}$ x $1197 \mathrm{~mm}$ x $32 \mathrm{~mm}$, relief léger. Ce tableau peut être vu au Tate Modern à Londres. Voir aussi http ://www.tate.org.uk/. 
On pourrait être tenté, plutôt naïvement, d'oser une lecture représentationnelle de l'œuvre : serait-ce la représentation du bleu en soi ? C'est ce que Klein lui-même a répondu aux «béotiens » qui lui posaient incessamment la question "mais qu'est-ce que ça représente ? " : ç̧a représente tout simplement du bleu en soi, disait-il ironiquement, ou du rouge en soi, ou bien c'est le paysage du monde de la couleur jaune ${ }^{27}$. " La couleur aurait alors une valeur expressive en soi. Rien dans l'œuvre ne s'oppose immédiatement à ce point de vue. Mais cette réponse facile à la question de la représentation ne tient pas la route, comme l'indique aussi la réponse d'Adorno devant cette possibilité (réponse avec laquelle Klein serait largement d'accord, bien entendu) : «La valeur expressive d'un rouge était déjà une illusion. [...] Réduit au matériau naturel, tout cela est vide, et les théorèmes qui servent à la mystification n'ont pas plus de substance que n'en a la charlatanerie des expériences tonales. Seul le récent physicalisme, par exemple en musique, effectue une réduction littérale à des éléments, [une forme de] spiritualisation qui, par suite, expulse l'esprit ${ }^{28}$.»

Manifestement, nous ne pouvons commencer à interpréter la couleur comme étant intrinsèquement signifiante et, par conséquent, susceptible d'être directement compréhensible. Le caractère moderne de l'œuvre constitue un point de départ plus intéressant: en raison de sa situation dans la tradition du modernisme et de l'abstraction, IKB 79 n'a pas trait à quelque chose d'objectif ou même de psychologique; il veut plutôt faire référence à l'apparence elle-même - possibilité intrinsèque de l'art qui surgit au premier plan une fois que la simple représentation (d'un contenu thématique) s'est avérée naïve ou impossible. Mais évidemment, que IKB 79 ait trait à l'apparence ne peut revenir à la thèse simplificatrice selon laquelle les œuvres non objectives mettent en évidence la difficulté de la représentation. Cette thèse, par exemple, ne nous procure pas de critères pour distinguer une œuvre de Pollock d'une œuvre de Klein, ce qui apparaît comme une conséquence plutôt gênante. IKB 79 de Klein gagne plutôt à être comprise comme ayant trait à l'apparence dans la mesure, précisément, où l'œuvre est liée à une tentative de nous présenter " la matière liberté impalpable » sous la forme qui lui est propre : la surface monochrome.

Comment entamer l'interprétation de IKB 79? Tout d'abord, il est possible de dire que le bleu breveté IKB de Klein est un bleu ultramarin qui combine du pigment et de la résine synthétique afin de l'appliquer sur le support. Il colore la surface du support (en l'occurrence, une plaque de bois) uniformément. La couleur semble adhérer à la surface de l'objet, le laissant tel quel et, pourtant, le transformant de manière englobante par l'application du pigment IKB, lequel, pour sa part, appartient conceptuellement à la subjectivité de l'artiste, tant aux sens créatif que légal. En outre, parce que l'œuvre est constituée uniquement de ce support et du pigment qui le recouvre, la

27. Klein, "L'aventure monochrome », p. 75.

28. Adorno, Théorie esthétique, p. 135. - GS, t. 7, p. 140. 
couleur IKB peut être vue à la fois comme étrangère au support et comme faisant tout aussi inextricablement partie de lui: l'absence de tout autre matériau nous montre que ce n'est qu'un support apprêté, mais également un support traité avec de l'IKB. Quant au support, il accepte passivement la couleur qui en devient partie intégrante. Par ces gestes, l'œuvre est produite. En effet, elle devient ce qu'elle est seulement grâce à la subsomption du caractère physique du support et du pigment dans la réalité spirituelle de la couleur IKB. L'œuvre est le résultat de ce processus et survient de l'application synthétique de cette substance totalement envahissante sur un objet tout à fait banal. Le monochrome dit: tout est un.

Si les choses en restaient là, la réaction d'Adorno serait tout à fait prévisible: "C'est justement l'insatiable principe d'identité qui éternise l'antagonisme en opprimant ce qui est contradictoire. Ce qui ne tolère rien qui ne soit pareil à lui-même contrecarre une réconciliation pour laquelle il se prend faussement. La violence du «rendre semblable» reproduit la contradiction qu'elle élimine ${ }^{29}$. " "L'insatiable principe d'identité », critiqué ici par Adorno, est bien entendu celui qui sous-tend la connaissance de manière générale; c'est le principe ultime non seulement de la pensée hégélienne, mais celui de tout savoir qui se base sur le sujet-connaissant et sa puissance générique de réduire tout à l'identité (celle du concept, du sujet) : «penser signifie identifier ${ }^{30}$. La critique adornienne de ce principe consiste, comme on le sait, à dénoncer la colonisation ou l'expulsion du non-identique qu'il implique : "Il faut faire opposition à la totalité en la convainquant de sa non-identité avec elle-même, nonidentité qu'elle nie de par son propre concept ${ }^{31}$.»

On pourrait dire, alors, que $I K B 79$ pose problème dans la mesure où il soutient la validité du principe d'identité par la réduction à un concept unique et totalisant, celui de la couleur IKB. En effet, IKB 79 semble figurer, dans un sens, la production même de l'objectivité effectuée par l'esprit, traduisant de cette façon le mouvement de l'esprit même - c'est-à-dire de la spiritualisation de la nature - comme sa véritable méthode et son contenu. Donc, IKB 79 prendrait pour sujet le travail de la cognition : il figure la compréhension subjective de l'objectivité par des concepts, en l'occurrence celui de l'IKB. Mais un problème se pose: comment Klein peut-il prétendre nous présenter la "matière liberté impalpable si, comme c'est le cas pour le principe « insatiable» d'identité, rien n'échappe au concept IKB ou si, en termes hégéliens, l'immédiateté est toujours déjà supprimée ${ }^{32}$ ? Comment la liberté se manifeste-

29. Theodor W. Adorno, Dialectique négative, trad. Groupe de traduction du Collège de philosophie (Paris: Payot, 2001), p. 142 . - GS, t. 6, p. 146.

30. Adorno, Dialectique négative, p. 15. GS, t. 6, p. 17. Selon Adorno, ce principe est à l'œuvre dans d'autres contextes aussi : ceux, par exemple, des sciences de la nature et de la production de biens culturels. Voir Max Horkheimer et Theodor W. Adorno, La dialectique de la raison: fragments philosophiques, trad. É. Kaufholz (Paris: Gallimard, 1974). Adorno, GS, t. 3.

31. Adorno, Dialectique négative, p. 146. - GS, t. 6, p. 150.

32. Ou encore, si le monochrome reproduit seulement « la nuit où toutes les vaches sont noires ". 
t-elle, et en quoi la matière est-elle impalpable ? Le pigment IKB et, de façon correspondante, le concept IKB semblent couvrir uniformément et totalement le support - rien n'est épargné ; tout a été réduit conformément au standard IKB. Comme Hegel l'aurait dit, l'esprit se réalise seulement dans la suppression de la nature. Le monochrome peut-il être sauvé du verdict d'Adorno?

Comme Klein lui-même le suggère, si $I K B 79$ réussit à présenter au regard la "matière liberté impalpable ", c'est précisément parce que la liberté ou l'ipséité de l'objet, son expressivité, demeure discernable malgré l'effet englobant de l'IKB. Dès lors, $I K B 79$ aurait trait à ce qui échappe à l'identité : l'ipséité (la matière liberté) qui se tient en réserve face à une identification conceptuelle particulière - sans pour autant devenir une chose en soi ou un immédiat qui se préserverait, sauf, de la cognition. S'il pouvait rencontrer ce critère, il pourrait alors réaliser une tension véritablement expressive entre les éléments minimaux de l'œuvre et, du coup, contrer la critique adornienne du monochrome. La méthode absolue de Klein ne se réduirait pas, dans ce cas, à la littéralité barbare critiquée par Adorno.

D'une part, IKB 79 reprend ou «imite » le mouvement du concept à travers la nature : il rend, préserve, et annule également l'immédiateté de l'être (ou son représentant, le support). Qui plus est, ce mouvement est doublé par un autre dans le pigment lui-même qui est aussi un matériau naturel spiritualisé (dans la mesure où l'IKB est une invention protégée). Effectivement, le concept IKB tient la place de l'esprit subjectif et, du coup, garantit que l'œuvre fait valoir sa matière spiritualisée et donc ne demeure pas un simple objet arbitraire: l'arbitraire est contré par la spécificité subjective indéniable de l'IKB en tant que couleur déjà spiritualisée (c'est-à-dire, personnalisée, brevetée) au lieu d'être un pigment purement banal et anonyme.

D'autre part, dans l'indispensable expérience esthétique de la couleur de l'œuvre, qui est loin d'être une simple composante arbitraire, une certaine non-identité résistant à la compréhension conceptuelle totalisante se profile : bien qu'il nomme la couleur de façon parfaitement adéquate, le concept IKB ne nous donne en aucune façon accès à la couleur «en soi ». La preuve en est qu'il est impossible d'expliquer les caractéristiques physiques et conceptuelles du pigment IKB à quelqu'un et de s'attendre à ce qu'il puisse, sur ces seules bases, connaître la couleur en question : la couleur doit être vue, expérimentée en tant qu'IKB ${ }^{33}$. Le concept en lui-même ne nous donne rien s'il n'est pas comblé par l'expérience du pigment IKB. Sans cette expérience, il n'y a pas d'œuvre : l'expérience vécue de la couleur est un de ses aspects irréductibles. Cette effectivité ultramarine est donc en fin de compte ce qui sauve les mono-

33. Hume parle d'une nuance inconnue de bleu qui peut néanmoins être discernée et suppléée par l'imagination. Mais il suppose un contexte qui sert à guider l'imagination: une gradation de nuances particulières de bleu qui ne comprend pas la nuance inconnue. Ce contexte ne nous est pas donné par l'IKB de Klein, dont la définition purement conceptuelle ou chimique ne peut évoquer sa réalité sensible. 
chromes de Klein de leur participation à une idée monstrueuse et vide, l'idéal monochrome privé de toute tension picturale interne : l'expérience réelle de la couleur est une conditio sine qua non d'une manifestation artistique quelconque du concept $\mathrm{IKB}^{34}$. Bien entendu, cette expérience ne vise pas une immédiateté non conceptuelle. La couleur remplit le concept, tout comme le concept définit la couleur. L'essentiel à cet égard est que l'expérience nécessaire de la couleur, le concept réalisé, ne résout aucun problème; au contraire, l'expérience concrète de $I K B 79$ nous pose un problème : est-ce de l'art ou non ? Voilà comment l'œuvre suscite l'interprétation à travers sa répétition de la cognition. Nous répondrons à cette question par le biais d'un bref résumé et d'un approfondissement des thèmes explorés plus haut.

L'IKB 79 est donc la transcription de la relation dialectique qui lie concept et chose sensible, pensée et être. Il "portraiture » la manière dont un concept (en l'occurrence, le concept IKB) nous livre la réalité conformément à un élan subjectif particulier sans la trahir. Parce que Klein laisse le support et le pigment tels quels, sans les encombrer "de lignes, de formes, de composition", il nous permet d'entrevoir la configuration de la chose «en soi » développée par le concept IKB. Le tableau nous offre la possibilité d'apprécier la matérialité pure par le biais d'une idéalité unique: les extrêmes se touchent.

Ce traitement monochrome de la matière est, en tant que processus, une spiritualisation en profondeur de la nature dont le résultat artistique réfère à ce processus même ainsi qu'à la préservation de la non-identité (la matière) vis-à-vis toute réduction au concept IKB. La critique adornienne, selon laquelle l'art qui ne va pas assez loin bredouille en raison du manque de tensions internes qui pourraient donner lieu à une sorte de cohérence logique, peut ainsi être parée. Loin de succomber à la réduction à l'insignifiance des matériaux bruts, la cohérence logique de $I K B 79$ peut être appréhendée en vertu précisément de cette caractéristique qui doit constituer le vrai art, à savoir, l'articulation signifiante des matériaux dans l'apparence; dans le cas de Klein, cela correspond à l'apparence sensible du travail de cognition. Donc, malgré la critique d'Adorno, IKB 79 de Klein se donne comme un « lieu où l'esprit artistique se hausse au-dessus du simple existant [ou encore, comme] l'idée qui ne capitule pas devant le simple étant-là des matériaux et des techniques $^{35}$ ». Ce que l'analyse nous montre c'est que «ce qui se passe dans l'œuvre, ce par quoi elle s'organise, ce par quoi elle se modifie ${ }^{36}$ " ne se réduit pas à une méthode absolue ou à une simple technique de production. L'œuvre agit en transformant par un acte délibéré de l'esprit subjectif ce qui serait autrement une simple existence ou une technique : l'application de ce bleu unique, irré-

34. Mais le concept est manifeste, dira-t-on, dans la formule chimique qui le décrit. Certes, mais l'IKB en tant que pigment et procédé d'application implique directement son utilisation et son effectivité dans la production d'œuvres d'art.

35. Adorno, Théorie esthétique, p. 64 . GS, t. 7, p. 63.

36. Adorno, Théorie esthétique, pp. 494. GS, t. 7, p. 529. 
ductiblement sensible et conceptuel. Cela nous permet désormais de voir l'intérêt particulier de Klein : les blancs de Rauschenberg et de Manzoni ne peuvent pas servir de substitut ici. Seul l'IKB de Klein, parce qu'il porte la marque de l'esprit subjectif en tant que processus breveté, assure à l'œuvre son caractère d'apparence authentique, qui est la tension non résolue entre l'esprit et la nature dans une signification esthétique énigmatique et ouverte. $I K B 79$ est en quelque sorte un "portrait de la cognition " sous sa forme subjective. Le blanc de Rauschenberg est un blanc comme n'importe quel autre blanc, alors que le bleu de Klein est déjà spiritualisé.

Évidemment, que l'IKB ait appartenu à Klein n'est pas l'essentiel. Son tour de force consiste plutôt à montrer que l'objectivité devient objective, connaissable, seulement dans son rapport à un processus subjectif d'appropriation $^{37}$. L'objet devient l'objet qu'il est seulement grâce à son développement conceptuel. Toutefois, ce développement, celui de l'application de l'IKB sur un support, est très particulier en cela qu'il laisse un reste, pour ainsi dire. L'IKB nous livre pleinement un objet dans l'expérience esthétique, mais seulement en suscitant davantage la cognition : l'œuvre est «ouverte » en ce sens qu'elle demande toujours à être interprétée à nouveau. L'IKB n'est donc pas un concept qui épuise son objet; il est plutôt un concept qui conduit forcément à d'autres, à l'interprétation, à l'apparence esthétique énigmatique qui pose une question à laquelle nos interprétations tentent de donner une réponse. L'IKB est, en ce sens, un concept qui défend la liberté de la matière par le geste même qui la spiritualise.

Donc si l'on parle de l'ipséité ou de la "matière liberté impalpable », c'est que l'on signale ainsi que l'œuvre se tient en réserve en se donnant à l'interprétation, non pas comme une immédiateté inconnaissable, mais plutôt comme un lieu d'interprétation ouvert. Bien entendu, l'interprète n'est pas pour autant autorisé à dire n'importe quoi : l'œuvre nous donne une piste ou une norme qui sert à guider la réflexion et à la contraindre, sans ne jamais communiquer une vérité quelconque "cachée » dans l'œuvre. La vérité de l'œuvre, si l'on peut parler ainsi, n'est rien d'autre que la découverte de cette normativité immanente (les "lois de son mouvement») qui nous contraint dans le développement même de nos interprétations. Donc, si «matière liberté impalpable » veut dire quelque chose, cela signifie : norme impalpable mais réelle, élaborée à partir de la matière de l'œuvre que nous interprétons sans jamais épuiser sa liberté devant nos tentatives d'interprétation. En ce sens, «matière liberté impalpable » est une définition possible du non-identique adornien qui nargue le principe d'identité et qui, dans $I K B 79$, transcende par conséquent le « $\mathrm{A}=\mathrm{A}$ » du monochrome raté (où le pigment et le support ne sont que pigment et support).

37. "Je veux créer des œuvres qui soient nature et esprit », dit-il. Voir Klein, "L'aventure monochrome », p. 83. 
Dès lors, parce que le caractère de l'apparence esthétique réside dans la tension entre nature et esprit, matérialité et idéalité, IKB 79 peut être accepté comme une œuvre authentique, en dépit des efforts d'Adorno pour nous convaincre de la vacuité de telles œuvres. De la sorte, IKB 79 se positionne ironiquement comme un exemple des intuitions les plus fondamentales d'Adorno concernant l'art moderne du XX $\mathrm{XX}^{\mathrm{e}}$ siècle; mieux encore, IKB 79 en ferait son sujet même! En répondant à l'apparence de l'œuvre et à son processus unique de spiritualisation, la pensée doit néanmoins fidèlement en observer les exigences et réactiver la mémoire de domination que la spiritualisation inscrit dans chaque œuvre, qui est d'ailleurs le sujet même de IKB 79; de cette façon, l'expérience esthétique projette un modèle de la connaissance qui incarne une forme plus douce de la raison que celle qui se révèle à travers la rationalité instrumentale de la société capitaliste tardive.

Interpréter l'art comme nous l'avons fait avec IKB 79 , ce n'est pas le déchiffrer et dévoiler son symbolisme caché. Interpréter une œuvre, c'est toujours plutôt en répondre par une quête de mots les plus justes, obéir à la normativité implicite dans son apparence unique, tout en sachant que ce geste s'accompagne d'une double condition : d'une part, en interprétant une œuvre, on doit avouer qu'il est tout à fait concevable que l'on se trompe; mais, d'autre part, il est tout aussi impossible d'être si juste qu'aucune interprétation supplémentaire ne soit possible. La plus heureuse des interprétations ne trouvera jamais confirmation de ses efforts auprès de $I K B 79$ : il reste muet, se tait dans l'intensité de sa couleur et demeure un signe dont le signifié est seulement suggéré ; le mieux que nous puissions espérer n'est peut-être qu'une interprétation convaincante ou pertinente «ici » et «maintenant ». C'est dire que l'interprétation de $I K B 79$ en tant que portrait de la cognition doit être jugée à la fois à l'aune de l'œuvre et de la communauté historique des interprètes. En dépit de son mutisme, l'apparence d'une œuvre d'art est traversée d'une normativité socialement médiatisée, à laquelle l'interprétation doit s'ajuster — c'est-à-dire une normativité inhérente à la réalité et à l'histoire de l'œuvre qui, si elle ne peut confirmer une fois pour toutes que nous visons juste, peut néanmoins nous aider à déterminer que nous sommes dans l'erreur :

La connaissance ne possède complètement aucun de ses objets. Elle ne doit pas susciter le fantasme d'un tout. Ainsi, la tâche d'une interprétation philosophique des œuvres d'art ne peut pas produire leur identité au moyen du concept, les absorber en lui ; l'œuvre se déploie cependant dans sa vérité à travers l'interprétation. [...] En principe, [l'interprétation philosophique] peut toujours faire fausse route; et seulement pour cette raison gagner quelque chose ${ }^{38}$.

38. Adorno, Dialectique négative, p. 146. GS, t. 6, p. 25. L'auteur tient à remercier chaleureusement M. Martel, J. Gaiger, N. Walker, D. Davies et O. Mathieu pour leurs diverses contributions à l'évolution de cet article. 